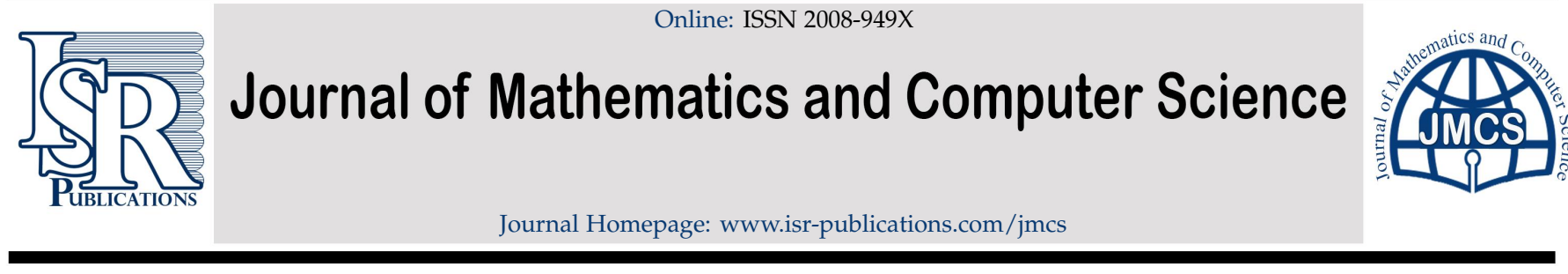

\title{
Nörlund statistical convergence and Tauberian conditions for statistical convergence from statistical summability us- ing Nörlund means in non-Archimedean fields
}

\author{
D. Eunice Jemima ${ }^{a}, *$, V. Srinivasan ${ }^{b}$ \\ ${ }^{a}$ Department of Mathematics, Faculty of Engineering and Technology, SRM Institute of Science and Technology, Kattankulathur, \\ Chennai-603203, India. \\ ${ }^{b}$ (Retd. Professor) Department of Mathematics, Faculty of Engineering and Technology, SRM Institute of Science and Technology, \\ Kattankulathur, Chennai-603203, India.
}

\begin{abstract}
In this paper, we define the concept of statistical convergence of sequences by Nörlund summability method and obtain a few results on the relationship between Nörlund summability and Nörlund statistical convergence in a complete, non-trivially valued, non-archimedean field K. Also, the necessary and sufficient Tauberian conditions under which statistical convergence follows from statistical summability by Nörlund means over $\mathrm{K}$ are discussed.
\end{abstract}

Keywords: Non-archimedean fields, Nörlund mean, statistical convergence, statistical summability $\left(N, p_{n}\right)$, Tauberian conditions.

2020 MSC: 40A35, 40E05, 40G05, 40G15, 46S10.

(C)2022 All rights reserved.

\section{Introduction}

In 1951, Fast [4] introduced the notion of statistical convergence. The relation between summability theory and statistical convergence was brought in by Schoenberg, which was later studied in detail by Fridy [5], Kolk, Freedman, Savas, Fridy and Miller [6], Mursaleen [10], Salat [12], Fridy and Orhan, Cakalli [3] etc. Monna [7] started a systematic study of Functional Analysis over a field other than the Real or Complex fields. A detailed study on the p-Adic numbers and Valuation theory was done by Bachman [1]. Suja and Srinivasan [14] introduced statistical convergence in non-archimedean fields.

Nörlund method of summability in non-archimedean fields was introduced by Srinivasan [13]. Natarajan [11] studied the relation between regular Nörlund methods and Nörlund summability. Braha [2], Fekete, Totur, Canak, Loku, etc. worked on Tauberian theorems using different methods of summability. Moricz [8] established the Tauberian conditions under which statistical convergence follows from statistical summability $(C, 1)$ and also by weighted means along with Orhan [9], in classical analysis. In this

\footnotetext{
*Corresponding author

Email addresses: eunicejem@gmail.com (D. Eunice Jemima), drvsrinivas.5@gmail.com (V. Srinivasan)

doi: $10.22436 /$ jmcs.024.04.02
}

Received: 2021-01-07 Revised: 2021-02-19 Accepted: 2021-02-21 
paper, the concept of statistical convergence of sequences by Nörlund summability method $\left(N, p_{n}\right)$ is defined, and a few results on the relation between $\left(N, p_{n}\right)$ summability and $\left(N, p_{n}\right)$ statistical convergence are found. Also, Tauberian conditions for sequences that are statistically summable by Nörlund means over non-archimedean fields are studied.

\subsection{Preliminaries}

Let $\mathrm{K}$ be a complete, non-trivially valued, non-archimedean field. (Recall that a valued field $(K,||$.$) is$ non-archimedean if $|a+b| \leqslant \max \{|a|,|b|\}$, for all $a, b \in K)$. A sequence $x=\left(x_{k}\right), x_{k} \in K, k=0,1,2, \cdots$ is said to be statistically convergent [14] to a limit ' $l$ ' if, for every $\epsilon>0$,

$$
\lim _{n \rightarrow \infty} \frac{1}{n}\left|\left\{k \leqslant n:\left|x_{k}-l\right| \geqslant \epsilon\right\}\right|=0,
$$

(where the outer vertical bars indicate the cardinality of the set), which we write as

$$
s t-\lim _{k \rightarrow \infty} x_{k}=l \text {. }
$$

Let $p=\left(p_{k}\right), k=0,1,2, \cdots$ be a sequence in $K$ such that $p_{0} \neq 0,\left|p_{0}\right|>\left|p_{j}\right|, j=1,2, \cdots$ and

$$
\mathrm{P}_{\mathrm{n}}=\sum_{\mathrm{k}=0}^{\mathrm{n}} \mathrm{p}_{\mathrm{k}}, \quad \mathrm{n}=0,1,2, \cdots .
$$

It is clear that $\left|P_{n}\right|=\left|p_{0}\right| \neq 0$, so $P_{n} \neq 0, n=0,1,2, \cdots$. Srinivasan [13] introduced the Nörlund method of summability, that is, the $\left(N, p_{n}\right)$ method in $K$ by the infinite matrix $\left(a_{n, k}\right)$ where

$$
a_{n, k}= \begin{cases}\frac{p_{n-k}}{P_{n}}, & k \leqslant n \\ 0, & k>n .\end{cases}
$$

Definition 1.1. The Nörlund mean $\left(N, p_{n}\right)$ of the sequence $x=\left(x_{n}\right)$ is defined by

$$
t_{n}=\frac{1}{P_{n}} \sum_{k=0}^{n} p_{n-k} x_{k}, \quad n=0,1,2, \cdots .
$$

Definition 1.2. The sequence $\left(x_{k}\right)$ is said to be statistically $\left(N, p_{n}\right)$ summable to a limit ' $l$ ' if

$$
s t-\lim _{n \rightarrow \infty} t_{n}=l \text {. }
$$

That is,

$$
\lim _{M \rightarrow \infty} \frac{1}{M}\left|\left\{n \leqslant M:\left|\frac{1}{P_{n}} \sum_{k=0}^{n} p_{n-k} x_{k}-l\right| \geqslant \epsilon\right\}\right|=0
$$

Definition 1.3. A sequence $x=\left(x_{k}\right)$ is said to be Nörlund statistically convergent to $l$ if, for every $\epsilon>0$

$$
\lim _{n \rightarrow \infty} \frac{1}{P_{n}}\left|\left\{k \leqslant n: p_{n-k}\left|x_{k}-l\right| \geqslant \epsilon\right\}\right|=0 .
$$

Definition 1.4. A sequence $x=\left(x_{k}\right)$ is said to be $\left(N, p_{n}\right)$ summable to $l$ if,

$$
\lim _{n \rightarrow \infty} \frac{1}{P_{n}} \sum_{k=0}^{n} p_{n-k}\left|x_{k}-l\right|=0 .
$$

Natarajan [11] proved that, if sequence $\left(x_{k}\right)$ is $\left(N, p_{n}\right)$ summable, then $\left(x_{k}\right)$ is bounded, and also proved the necessary and sufficient conditions for a regular $\left(N, p_{n}\right)$ method, stated in the definition below.

Definition 1.5. The $\left(N, p_{n}\right)$ method is regular if and only if $p_{n} \rightarrow 0$ as $n \rightarrow \infty$.

In this section, we consider the $\left(N, p_{n}\right)$ method to be regular. 


\section{New results}

Theorem 2.1. Let $\frac{P_{n}}{n}>1$, for every $n \in \mathbb{N}$. If $\left(x_{k}\right)$ is statistically convergent to $l$, then $\left(x_{k}\right)$ is statistically $\left(\mathrm{N}, \mathrm{p}_{\mathrm{n}}\right)$ convergent to $\mathrm{l}$.

Proof. Given, $\left(x_{k}\right)$ is statistically convergent to $l$. That is,

$$
\lim _{n \rightarrow \infty} \frac{1}{n}\left|\left\{k \leqslant n:\left|x_{k}-l\right| \geqslant \epsilon\right\}\right|=0 .
$$

To prove $\left(x_{k}\right)$ is statistically $\left(N, p_{n}\right)$ convergent to $l$, that is to prove

$$
\lim _{n \rightarrow \infty} \frac{1}{P_{n}}\left|\left\{k \leqslant n: p_{n-k}\left|x_{k}-l\right| \geqslant \epsilon\right\}\right|=0,
$$

consider

$$
\begin{aligned}
\frac{1}{P_{n}}\left|\left\{k \leqslant n: p_{n-k}\left|x_{k}-l\right| \geqslant \epsilon\right\}\right| & =\frac{n}{P_{n}} \times \frac{1}{n}\left|\left\{k \leqslant n: p_{n-k}\left|x_{k}-l\right| \geqslant \epsilon\right\}\right| \\
& \leqslant \frac{1}{n}\left|\left\{k \leqslant n: p_{n-k}\left|x_{k}-l\right| \geqslant \epsilon\right\}\right| \quad\left(\text { since } \frac{n}{P_{n}}<1\right) \\
& \rightarrow 0, \text { as } n \rightarrow \infty . \quad\left(\text { since } p_{n} \rightarrow 0, n \rightarrow \infty\right. \text { and by (2.1)) }
\end{aligned}
$$

Therefore,

$$
\lim _{n \rightarrow \infty} \frac{1}{P_{n}}\left|\left\{k \leqslant n: p_{n-k}\left|x_{k}-l\right| \geqslant \epsilon\right\}\right|=0,
$$

or, $\left(x_{k}\right)$ is statistically $\left(N, p_{n}\right)$ convergent to $l$.

The following example is an illustration of this theorem.

Example 2.2. Consider the sequence $x=\left(x_{k}\right)$ defined by

$$
x_{k}= \begin{cases}\frac{k-1}{k^{2}+1}, & \text { if } k \text { is a perfect square, } \\ 0, & \text { otherwise. }\end{cases}
$$

Choosing the non-archimedean valuation to be 2-adic, the terms of the sequence are

$$
\left(0,0,0,1,0,0,0,0, \frac{1}{4}, 0,0, \cdots\right) .
$$

This sequence is clearly statistically convergent to 0 , since,

$$
\lim _{n \rightarrow \infty} \frac{1}{n}\left|\left\{k \leqslant n:\left|x_{k}-0\right| \geqslant \epsilon\right\}\right|=0 .
$$

Let $\left(p_{n}\right)=\left(3^{n}\right), n=0,1,2, \cdots$ be a $\left(N, p_{n}\right)$ method in the 2-adic field $Q_{2}$. Then, $\left(p_{n}\right)=(1,1,1, \cdots)$. Therefore,

$$
\begin{aligned}
\mathrm{P}_{\mathrm{n}} & =\mathrm{p}_{0}+\mathrm{p}_{1}+\cdots+\mathrm{p}_{\mathrm{n}} \\
& =1+1+\cdots+1 \\
& =|\mathrm{n}+1|_{2} .
\end{aligned}
$$

Now,

$$
\lim _{n \rightarrow \infty} \frac{1}{P_{n}}\left|\left\{k \leqslant n: p_{n-k}\left|x_{k}-0\right| \geqslant \epsilon\right\}\right|=\lim _{n \rightarrow \infty} \frac{1}{|n+1|_{2}}\left|\left\{k \leqslant n: 3^{n-k}\left|x_{k}\right| \geqslant \epsilon\right\}\right|=0,
$$

which shows that $\left(x_{k}\right)$ is statistically $\left(N, p_{n}\right)$ convergent to 0 . 
Theorem 2.3. If the sequence $\left(P_{n}\right)$ is bounded such that $\lim _{n \rightarrow \infty} \sup \frac{P_{n}}{n}<\infty$, and if $\left(x_{k}\right)$ is statistically $\left(N, p_{n}\right)$ convergent to $l$, then $\left(x_{k}\right)$ is statistically convergent to $l$.

Proof. Given, $\left(x_{k}\right)$ is statistically $\left(N, p_{n}\right)$ convergent to $l$; that is,

$$
\lim _{n \rightarrow \infty} \frac{1}{P_{n}}\left|\left\{k \leqslant n: p_{n-k}\left|x_{k}-l\right| \geqslant \epsilon\right\}\right|=0 .
$$

To prove $\left(x_{k}\right)$ is statistically convergent to $l$; that is, to prove

$$
\lim _{n \rightarrow \infty} \frac{1}{n}\left|\left\{k \leqslant n:\left|x_{k}-l\right| \geqslant \epsilon\right\}\right|=0,
$$

consider

$$
\begin{aligned}
\frac{1}{n}\left|\left\{k \leqslant n:\left|x_{k}-l\right| \geqslant \epsilon\right\}\right| & \leqslant \frac{1}{n}\left|\left\{k \leqslant n: p_{n-k}\left|x_{k}-l\right| \geqslant \epsilon\right\}\right| \\
& \leqslant \frac{P_{n}}{n} \times \frac{1}{P_{n}}\left|\left\{k \leqslant n: p_{n-k}\left|x_{k}-l\right| \geqslant \epsilon\right\}\right| \\
& \left.\leqslant \frac{1}{P_{n}}\left|\left\{k \leqslant n: p_{n-k}\left|x_{k}-l\right| \geqslant \epsilon\right\}\right| \quad \text { (since } \lim _{n \rightarrow \infty} \sup \frac{P_{n}}{n}<\infty\right) \\
& \rightarrow 0 \text { as } n \rightarrow \infty \quad(\text { by }(2.2)) .
\end{aligned}
$$

Thus, $\left(x_{k}\right)$ is statistically convergent to $l$.

Theorem 2.4. If the sequence $\left(\mathrm{x}_{\mathrm{k}}\right)$ is $\left(\mathrm{N}, \mathrm{p}_{\mathrm{n}}\right)$ summable to $\mathrm{l}$, then $\left(\mathrm{x}_{\mathrm{k}}\right)$ is statistically $\left(\mathrm{N}, \mathrm{p}_{\mathrm{n}}\right)$ convergent to $\mathrm{l}$. Proof. Given, $\lim _{n \rightarrow \infty} t_{n}=l$. That is,

$$
\lim _{n \rightarrow \infty} \frac{1}{P_{n}} \sum_{k=0}^{n} p_{n-k} x_{k}=l,
$$

i.e.,

$$
\lim _{n \rightarrow \infty}\left(p_{n} x_{0}+p_{n-1} x_{1}+\cdots+p_{0} x_{n}\right)=\lim _{n \rightarrow \infty} P_{n} l=\lim _{n \rightarrow \infty}\left(p_{0}+p_{1}+\cdots+p_{n}\right) l
$$

i.e.,

$$
\lim _{n \rightarrow \infty}\left[p_{n}\left(x_{0}-l\right)+p_{n-1}\left(x_{1}-l\right)+\cdots+p_{0}\left(x_{n}-l\right)\right]=0,
$$

i.e.,

$$
\lim _{n \rightarrow \infty}\left|p_{n}\left(x_{0}-l\right)+p_{n-1}\left(x_{1}-l\right)+\cdots+p_{0}\left(x_{n}-l\right)\right|=0,
$$

which implies that

$$
\lim _{n \rightarrow \infty} \max \left\{\left|p_{n}\left(x_{0}-l\right)\right|,\left|p_{n-1}\left(x_{1}-l\right)\right|, \cdots,\left|p_{0}\left(x_{n}-l\right)\right|\right\}=0 .
$$

That is,

$$
\lim _{n \rightarrow \infty}\left|p_{n-k}\right|\left|x_{k}-l\right|=0, \quad k=0,1, \cdots, n,
$$

implies,

$$
\lim _{n \rightarrow \infty}\left|\left\{k \leqslant n: p_{n-k}\left|x_{k}-l\right| \geqslant \epsilon\right\}\right|=0,
$$

or,

$$
\lim _{n \rightarrow \infty} \frac{1}{P_{n}}\left|\left\{k \leqslant n: p_{n-k}\left|x_{k}-l\right| \geqslant \epsilon\right\}\right|=0 .
$$

This proves that $\left(x_{k}\right)$ is statistically $\left(N, p_{n}\right)$ convergent to $l$. 
Theorem 2.5. If $\left(x_{k}\right)$ is statistically $\left(N, p_{n}\right)$ convergent to $l$, then $\left(x_{k}\right)$ is $\left(N, p_{n}\right)$ summable to $l$.

Proof. Given,

$$
\lim _{n \rightarrow \infty} \frac{1}{P_{n}}\left|\left\{k \leqslant n: p_{n-k}\left|x_{k}-l\right| \geqslant \epsilon\right\}\right|=0 .
$$

Let us assume the contrary that $\left(x_{k}\right)$ is not $\left(N, p_{n}\right)$ summable to $l$. That is,

$$
\lim _{n \rightarrow \infty} \frac{1}{P_{n}} \sum_{k=0}^{n} p_{n-k} x_{k}>l \text {. }
$$

i.e.,

$$
\begin{aligned}
\lim _{n \rightarrow \infty}\left(p_{n} x_{0}+p_{n-1} x_{1}+\cdots+p_{0} x_{n}\right) & >\lim _{n \rightarrow \infty} p_{n} l \\
& >\lim _{n \rightarrow \infty}\left(p_{0}+p_{1}+\cdots+p_{n}\right) l,
\end{aligned}
$$

i.e.,

$$
\lim _{n \rightarrow \infty}\left[p_{n}\left(x_{0}-l\right)+p_{n-1}\left(x_{1}-l\right)+\cdots+p_{0}\left(x_{n}-l\right)\right]>0,
$$

implies,

$$
\lim _{n \rightarrow \infty}\left|p_{n}\left(x_{0}-l\right)+p_{n-1}\left(x_{1}-l\right)+\cdots+p_{0}\left(x_{n}-l\right)\right|>0,
$$

which further implies that

$$
\lim _{n \rightarrow \infty} \max \left\{\left|p_{n}\left(x_{0}-l\right)\right|,\left|p_{n-1}\left(x_{1}-l\right)\right|, \cdots,\left|p_{0}\left(x_{n}-l\right)\right|\right\}>0,
$$

or,

$$
\lim _{n \rightarrow \infty}\left|p_{n-k}\right|\left|x_{k}-l\right|>0, \quad k=0,1, \cdots, n
$$

implies,

$$
\lim _{n \rightarrow \infty}\left|\left\{k \leqslant n: p_{n-k}\left|x_{k}-l\right| \geqslant \epsilon\right\}\right|>0 .
$$

Also,

$$
\lim _{n \rightarrow \infty} \frac{1}{P_{n}}\left|\left\{k \leqslant n: p_{n-k}\left|x_{k}-l\right| \geqslant \epsilon\right\}\right|>0 .
$$

But this cannot happen by (2.3). Thus, $\left(x_{k}\right)$ is $\left(N, p_{n}\right)$ summable to $l$.

This theorem is illustrated by the following example.

Example 2.6. For the sequence $x=\left(x_{k}\right)$ together with the sequence $\left(p_{k}\right)$ and the 2 -adic valuation discussed in the previous example, which is statistically $\left(N, p_{n}\right)$ convergent to 0 , we have

$$
\lim _{n \rightarrow \infty} \frac{1}{P_{n}} \sum_{k=0}^{n} p_{n-k}\left|x_{k}-0\right|=\lim _{n \rightarrow \infty} \frac{1}{|n+1|_{2}} \sum_{k=0}^{n} 3^{n-k}\left|x_{k}\right|=0 .
$$

Thus it is clear that $\left(x_{k}\right)$ is $\left(N, p_{n}\right)$ summable to 0 .

Theorem 2.7. Let $\mathrm{p}=\left(\mathrm{p}_{\mathrm{k}}\right)$ be a sequence in $\mathrm{K}$ such that $\mathrm{p}_{0} \neq 0,\left|\mathrm{p}_{0}\right|>\left|\mathrm{p}_{\mathrm{j}}\right|, j=1,2, \cdots$. Let $\left(\lambda_{\mathrm{k}}\right)$ be a sequence in $\mathrm{K}$ such that $\lim _{\mathrm{k} \rightarrow \infty} \lambda_{\mathrm{k}}=0$ and

$$
\text { st }-\lim _{n \rightarrow \infty} \frac{P_{n}}{P_{\lambda_{n}}}<1, \text { for every } 0<\lambda_{n}<1 .
$$

Let $\mathrm{x}=\left(\mathrm{x}_{\mathrm{k}}\right), \mathrm{x}_{\mathrm{k}} \in \mathrm{K}, \mathrm{k}=0,1,2, \cdots$, be a sequence which is statistically $\left(\mathrm{N}, \mathrm{p}_{\mathrm{n}}\right)$ summable to a limit $\mathrm{l}$. Then $\left(\mathrm{x}_{\mathrm{k}}\right)$ is statistically convergent to $l$ if and only if for every $\epsilon>0$,

$$
\lim _{M \rightarrow \infty} \frac{1}{M}\left|\left\{n \leqslant M:\left|\frac{1}{\left(P_{n}-P_{\lambda_{n}}\right)} \sum_{k=\lambda_{n}+1}^{n} p_{n-k}\left(x_{n}-x_{k}\right)\right| \geqslant \epsilon\right\}\right|=0 .
$$


The following Lemmas are required in proving the theorem.

Lemma 2.8. Let $\mathrm{p}=\left(\mathrm{p}_{\mathrm{k}}\right)$ be a sequence in $\mathrm{K}$ such that $\mathrm{p}_{0} \neq 0,\left|\mathrm{p}_{0}\right|>\left|\mathrm{p}_{\mathrm{j}}\right|, j=1,2, \cdots$, and

$$
\text { st }-\lim _{n \rightarrow \infty} \frac{P_{n}}{P_{\lambda_{n}}}<1, \text { for every } 0<\lambda_{n}<1 \text {, }
$$

where $\left\{\lambda_{k}\right\}$ is a sequence in $\mathrm{K}$ such that $\lim _{\mathrm{k} \rightarrow \infty} \lambda_{k}=0$. Let $\mathrm{x}=\left(\mathrm{x}_{\mathrm{k}}\right), \mathrm{x}_{\mathrm{k}} \in \mathrm{K}, \mathrm{k}=0,1,2, \cdots$ be a sequence which is statistically $\left(N, p_{n}\right)$ summable to a limit $l$. Then for every $0<\lambda_{n}<1$,

$$
\text { st }-\lim _{n \rightarrow \infty} t_{\lambda_{n}}=l,
$$

where $\left(\mathrm{P}_{\mathrm{n}}\right)$ and $\left(\mathrm{t}_{\lambda_{\mathrm{n}}}\right)$ are non-decreasing sequences.

Proof. Given that the sequence $\left(x_{n}\right)$ is statistically $\left(N, p_{n}\right)$ summable to a limit $l$. This means that

$$
s t-\lim _{n \rightarrow \infty} t_{n}=l \text {. }
$$

That is

or,

$$
\lim _{M \rightarrow \infty} \frac{1}{M}\left|\left\{n \leqslant M:\left|t_{n}-l\right| \geqslant \epsilon\right\}\right|=0,
$$

$$
\lim _{M \rightarrow \infty} \frac{1}{M}\left|\left\{n \leqslant M:\left|\frac{1}{P_{n}} \sum_{k=0}^{n} p_{n-k} x_{k}-l\right| \geqslant \epsilon\right\}\right|=0 .
$$

To prove, st $-\lim _{n \rightarrow \infty} t_{\lambda_{n}}=l$, that is to prove

$$
\lim _{M \rightarrow \infty} \frac{1}{M}\left|\left\{\lambda_{n} \leqslant M:\left|t_{\lambda_{n}}-l\right| \geqslant \epsilon\right\}\right|=0,
$$

(or) to prove

$$
\lim _{M \rightarrow \infty} \frac{1}{M}\left|\left\{\lambda_{n} \leqslant M:\left|\frac{1}{P_{\lambda_{n}}} \sum_{k=0}^{\lambda_{n}} p_{\lambda_{n}-k} x_{k}-l\right| \geqslant \epsilon\right\}\right|=0
$$

let us consider

$$
\begin{aligned}
\frac{1}{M}\left|\left\{\lambda_{n} \leqslant M:\left|\frac{1}{P_{\lambda_{n}}} \sum_{k=0}^{\lambda_{n}} p_{\lambda_{n}-k} x_{k}-l\right| \geqslant \epsilon\right\}\right| & =\frac{1}{M}\left|\left\{\lambda_{n} \leqslant M:\left|\left(\frac{P_{n}}{P_{\lambda_{n}}}\right) \frac{1}{P_{n}} \sum_{k=0}^{\lambda_{n}} p_{\lambda_{n}-k} x_{k}-l\right| \geqslant \epsilon\right\}\right| \\
& \leqslant \frac{1}{M}\left|\left\{n \leqslant M:\left|\frac{1}{P_{n}} \sum_{k=0}^{n} p_{n-k} x_{k}-l\right| \geqslant \epsilon\right\}\right| \text { (using (2.4)) } \\
& \rightarrow 0 \text { as } M \rightarrow \infty . \quad \text { (using (2.6)) }
\end{aligned}
$$

Therefore,

$$
\lim _{M \rightarrow \infty} \frac{1}{M}\left|\left\{\lambda_{n} \leqslant M:\left|\frac{1}{P_{\lambda_{n}}} \sum_{k=0}^{\lambda_{n}} p_{\lambda_{n}-k} x_{k}-l\right| \geqslant \epsilon\right\}\right|=0,
$$

which shows that $s t-\lim _{n \rightarrow \infty} t_{\lambda_{n}}=l$. This proves the lemma.

We shall now prove,

Lemma 2.9. For $0<\lambda_{n}<1$,

$$
\frac{1}{\left(P_{n}-P_{\lambda_{n}}\right)} \sum_{k=\lambda_{n}+1}^{n} p_{n-k} x_{k}=t_{n}+\frac{P_{\lambda_{n}}}{\left(P_{n}-P_{\lambda_{n}}\right)}\left(t_{n}-t_{\lambda_{n}}\right),
$$

provided $\mathrm{P}_{\mathrm{n}}>\mathrm{P}_{\lambda_{\mathrm{n}}}$. 
Proof. Consider the right-hand side:

$$
\begin{aligned}
t_{n}+ & \frac{P_{\lambda_{n}}}{P_{n}-P_{\lambda_{n}}}\left(t_{n}-t_{\lambda_{n}}\right) \\
& =\frac{P_{n} t_{n}-P_{\lambda_{n}} t_{n}+P_{\lambda_{n}} t_{n}-P_{\lambda_{n}} t_{\lambda_{n}}}{P_{n}-P_{\lambda_{n}}} \\
& =\frac{1}{P_{n}-P_{\lambda_{n}}}\left[P_{n}\left(\frac{1}{P_{n}} \sum_{k=0}^{n} p_{n-k} x_{k}\right)-P_{\lambda_{n}}\left(\frac{1}{P_{\lambda_{n}}} \sum_{k=0}^{\lambda_{n}} p_{\lambda_{n}-k} x_{k}\right)\right] \\
& =\frac{1}{P_{n}-P_{\lambda_{n}}}\left[\sum_{k=0}^{\lambda_{n}} p_{\lambda_{n}-k} x_{k}+\sum_{k=\lambda_{n}+1}^{n} p_{n-k} x_{k}-\sum_{k=0}^{\lambda_{n}} p_{\lambda_{n}-k} x_{k}\right] \\
& =\frac{1}{P_{n}-P_{\lambda_{n}}} \sum_{k=\lambda_{n}+1}^{n} p_{n-k} x_{k} .
\end{aligned}
$$

Thus,

$$
\frac{1}{\left(P_{n}-P_{\lambda_{n}}\right)} \sum_{k=\lambda_{n}+1}^{n} p_{n-k} x_{k}=t_{n}+\frac{P_{\lambda_{n}}}{P_{n}-P_{\lambda_{n}}}\left(t_{n}-t_{\lambda_{n}}\right) .
$$

Now, adding $x_{n}$ to the above equation we get,

$$
x_{n}-t_{n}=\frac{P_{\lambda_{n}}}{P_{n}-P_{\lambda_{n}}}\left(t_{n}-t_{\lambda_{n}}\right)+\frac{1}{\left(P_{n}-P_{\lambda_{n}}\right)} \sum_{k=\lambda_{n}+1}^{n} p_{n-k}\left(x_{n}-x_{k}\right) .
$$

Proof of Theorem 2.7. Necessity: Here, we assume that

$$
s t-\lim _{n \rightarrow \infty} x_{n}=l,
$$

and prove that, for every $0<\lambda_{n}<1$,

$$
\lim _{M \rightarrow \infty} \frac{1}{M}\left|\left\{n \leqslant M:\left|\frac{1}{\left(P_{n}-P_{\lambda_{n}}\right)} \sum_{k=\lambda_{n}+1}^{n} p_{n-k}\left(x_{n}-x_{k}\right)\right| \geqslant \epsilon\right\}\right|=0 .
$$

Now, since st $-\lim _{n \rightarrow \infty} x_{n}=l$ and st $-\lim _{n \rightarrow \infty} t_{n}=l$, we have

$$
s t-\lim _{n \rightarrow \infty}\left(x_{n}-t_{n}\right)=0 .
$$

That is,

$$
\lim _{M \rightarrow \infty} \frac{1}{M}\left|\left\{n \leqslant M:\left|x_{n}-t_{n}\right| \geqslant \epsilon\right\}\right|=0 .
$$

This shows that

$$
\begin{aligned}
\lim _{M \rightarrow \infty} \frac{1}{M} \mid\left\{n \leqslant M: \mid \frac{P_{\lambda_{n}}}{\left(P_{n}-P_{\lambda_{n}}\right)}\left(t_{n}-t_{\lambda_{n}}\right)\right. & \\
& \left.+\frac{1}{\left(P_{n}-P_{\lambda_{n}}\right)} \sum_{k=\lambda_{n}+1}^{n} p_{n-k}\left(x_{n}-x_{k}\right) \mid \geqslant \epsilon\right\} \mid=0 . \quad \text { (using (2.7)) }
\end{aligned}
$$


Since the valuation is non-archimedean wherein $|a+b|=|a|$ if $|a|>|b|$, and since

$$
\frac{1}{M}\left|\left\{n \leqslant M:\left|\frac{P_{\lambda_{n}}}{\left(P_{n}-P_{\lambda_{n}}\right)}\left(t_{n}-t_{\lambda_{n}}\right)\right| \geqslant \epsilon\right\}\right| \rightarrow 0 \text { as } M \rightarrow \infty,
$$

by (1.1) and (2.5), we have that

$$
\lim _{M \rightarrow \infty} \frac{1}{M}\left|\left\{n \leqslant M:\left|\frac{1}{\left(P_{n}-P_{\lambda_{n}}\right)} \sum_{k=\lambda_{n}+1}^{n} p_{n-k}\left(x_{n}-x_{k}\right)\right| \geqslant \epsilon\right\}\right|=0 .
$$

Sufficiency: We now assume that

$$
\lim _{M \rightarrow \infty} \frac{1}{M}\left|\left\{n \leqslant M:\left|\frac{1}{\left(P_{n}-P_{\lambda_{n}}\right)} \sum_{k=\lambda_{n}+1}^{n} p_{n-k}\left(x_{n}-x_{k}\right)\right| \geqslant \epsilon\right\}\right|=0,
$$

and prove that

$$
s t-\lim _{n \rightarrow \infty} x_{n}=l
$$

To this end, it is enough if we prove that

$$
s t-\lim _{n \rightarrow \infty}\left(x_{n}-t_{n}\right)=0 .
$$

That is to prove,

$$
\lim _{M \rightarrow \infty} \frac{1}{M}\left|\left\{n \leqslant M:\left|x_{n}-t_{n}\right| \geqslant \epsilon\right\}\right|=0 .
$$

Using (2.7) we have,

$$
\begin{aligned}
\frac{1}{M}\left|\left\{n \leqslant M:\left|x_{n}-t_{n}\right| \geqslant \epsilon\right\}\right|= & \frac{1}{M} \mid\left\{n \leqslant M: \mid \frac{P_{\lambda_{n}}}{\left(P_{n}-P_{\lambda_{n}}\right)}\left(t_{n}-t_{\lambda_{n}}\right)\right. \\
& \left.+\frac{1}{\left(P_{n}-P_{\lambda_{n}}\right)} \sum_{k=\lambda_{n}+1}^{n} p_{n-k}\left(x_{n}-x_{k}\right) \mid \geqslant \epsilon\right\} \mid \\
\leqslant & \max \left\{\begin{array}{c}
\frac{1}{M}\left|\left\{n \leqslant M:\left|\frac{P_{\lambda_{n}}}{\left(P_{n}-P_{\lambda_{n}}\right)}\left(t_{n}-t_{\lambda_{n}}\right)\right| \geqslant \epsilon\right\}\right|, \\
\frac{1}{M}\left|\left\{n \leqslant M:\left|\frac{1}{\left(P_{n}-P_{\lambda_{n}}\right)} \sum_{k=\lambda_{n}+1}^{n} p_{n-k}\left(x_{n}-x_{k}\right)\right| \geqslant \epsilon\right\}\right|
\end{array}\right\} .
\end{aligned}
$$

By our assumption,

$$
\frac{1}{M}\left|\left\{n \leqslant M:\left|\frac{1}{\left(P_{n}-P_{\lambda_{n}}\right)} \sum_{k=\lambda_{n}+1}^{n} p_{n-k}\left(x_{n}-x_{k}\right)\right| \geqslant \epsilon\right\}\right| \rightarrow 0 \text { as } M \rightarrow \infty .
$$

Therefore,

$$
\begin{aligned}
\frac{1}{M}\left|\left\{n \leqslant M:\left|x_{n}-t_{n}\right| \geqslant \epsilon\right\}\right| & \leqslant \max \left\{\frac{1}{M}\left|\left\{n \leqslant M:\left|\frac{P_{\lambda_{n}}}{\left(P_{n}-P_{\lambda_{n}}\right)}\left(t_{n}-t_{\lambda_{n}}\right)\right| \geqslant \epsilon\right\}\right|, 0\right\} \\
& \leqslant \frac{1}{M}\left|\left\{n \leqslant M:\left|\frac{P_{\lambda_{n}}}{\left(P_{n}-P_{\lambda_{n}}\right)}\left(t_{n}-t_{\lambda_{n}}\right)\right| \geqslant \epsilon\right\}\right| \\
& \rightarrow 0 \text { as } M \rightarrow \infty, \quad \text { (by (1.1) and (2.5)) }
\end{aligned}
$$


which implies that

$$
\lim _{M \rightarrow \infty} \frac{1}{M}\left|\left\{n \leqslant M:\left|x_{n}-t_{n}\right| \geqslant \epsilon\right\}\right|=0,
$$

which means that

$$
\text { st }-\lim _{n \rightarrow \infty}\left(x_{n}-t_{n}\right)=0 .
$$

Thus, sequence $\left(x_{n}\right)$ is statistically convergent to ' $l$ '. This completes the proof of the theorem.

\section{Acknowledgment}

I am truly grateful to my Research supervisor Prof. Srinivasan Vaithinathasamy for his insightful comments. His knowledge, expertise and exacting attention to detail brought this article to its final form.

\section{References}

[1] G. Bachman, Introduction to p-Adic Numbers and Valuation Theory, Academic Press, New York-London, (1964). 1

[2] N. L. Braha, A Tauberian theorem for the generalized Nörlund-Euler summability method, J. Inequal. Spec. Funct., 7 (2016), 137-142. 1

[3] H. Çakallı, A Study on statistical convergence, Funct. Anal. Approx. Comput., 1 (2009), 19-24. 1

[4] H. Fast, Sur la convergence statistique, Colloq. Math., 2 (1951), 241-244. 1

[5] J. A. Fridy, On statistical Convergence, Analysis, 5 (1985), 301-313. 1

[6] J. A. Fridy, H. I. Miller, A matrix characterization of statistical convergence, Analysis, 11 (1991), 59-66. 1

[7] A. F. Monna, Sur le theoreme de Banach-Steinhaus, Nederl. Akad. Wetensch. Proc. Ser. A 66=Indag. Math., 25 (1963), 121-131. 1

[8] F. Moricz, Tauberian conditions under which statistical convergence follows from statistical summability $(\mathrm{C}, 1), \mathrm{J}$. Math. Anal. Appl., 275 (2002), 277-287. 1

[9] F. Moricz, C. Orhan, Tauberian conditions under which statistical convergence follows from statistical summability by weighted means, Studia Sci. Math. Hungar., 41 (2004), 391-403. 1

[10] M. Mursaleen, O. H. H. Edely, Generalized statistical convergence, Inform. Sci., 162 (2004), 287-294. 1

[11] P. N. Natarajan, On Nörlund method of summability in non-archimedean fields, J. Anal., 2 (1994), 97-102. 1, 1.1

[12] T. Salát, On statistically convergent sequences of real numbers, Math. Slovaca, 30 (1980), 139-150. 1

[13] V. K. Srinivasan, On certain summation processes in the p-adic field, Nederl. Akad. Wetensch. Proc. Ser. A 68=Indag. Math., 27 (1965), 319-325. 1, 1.1

[14] K. Suja, V. Srinivasan, On statistically convergent and statistically cauchy sequences in non-archimedean fields, J. Adv. Math., 6 (2014), 1038-1043. 1, 1.1 